\title{
CONF-950905--53
}

\section{A NEW TECHNIQUE TO MEASURE THE NEUTRALIZER CELL GAS LINE DENSITY APPLIED TO A DIII-D NEUTRAL BEAMLINE}

by

D.N. KESSLER, R.-M. HONG, and S.P. RIGGS

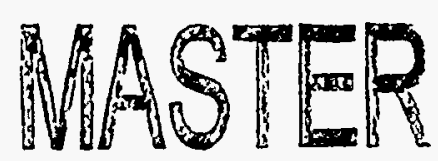

RECEIVED

APR 14 199?

OSTI

DSTRE

OCTOBER 1995 


\section{DISCLAIMER}

This report was prepared as an account of work sponsored by an agency of the United States Government. Neither the United States Government nor any agency thereof, nor any of their employees, makes any warranty, express or implied, or assumes any legal liability or responsibility for the accuracy, completeness, or usefulness of any information, apparatus, product, or process disclosed, or represents that its use would not infringe privately owned rights. Reference herein to any specific commercial product, process, or service by trade name, trademark, manufacturer, or otherwise, does not necessarily constitute or imply its endorsement, recommendation, or favoring by the United States Government or any agency thereof. The views and opinions of authors expressed herein do not necessarily state or reflect those of the United States Government or any agency thereof. 


\section{DISCLAIMER}

Portions of this document may be illegible in electronic image products. Images are produced from the best available original document. 


\title{
A NEW TECHNIQUE TO MEASURE THE NEUTRALIZER CELL GAS LINE DENSITY APPLIED TO A DIII-D NEUTRAL BEAMLINE
}

\author{
by \\ D.N. KESSLER, R.-M. HONG, and S.P. RIGGS
}

This is a preprint of a paper presented at the 16th IEEE/NPSS Symposium on Fusion Engineering, September 30-October 5, 1995, Champaign, Illinois, and to be printed in the Proceedings.

\author{
Work supported by \\ U.S. Department of Energy \\ Contract DE-AC03-89ER51114
}

\section{GENERAL ATOMICS PROJECT 3466 OCTOBER 1995}




\title{
A New Technique to Measure the Neutralizer Cell Gas Line Density Applied to a DIII-D Neutral Beamline*
}

\author{
D.N. Kessler, R.-M. Hong, S.P. Riggs \\ General Atomics \\ P.O. Box 85608, San Diego, California 92186-9784
}

\section{ABSTRACT}

The DIII-D tokamak employs eight ion sources for plasma heating. In order to obtain the maximum neutralization of energetic ions (providing maximum neutral beam power) and reduce the heat load on beamline internal components caused by residual energetic ions, sufficient neutral gas must be injected into the beamline neutralizer cell. The neutral gas flow rate must be optimized, however, since excessive gas will increase power losses due to neutral beam scattering and reionization.

It is important, therefore, to be able to determine the neutralizer cell gas line density. A new technique which uses the ion source suppressor grid current to obtain the neutralizer cell gas line density has been developed. The technique uses the fact that slow ions produced by beam-gas interactions in the neutralizer cell during beam extraction are attracted to the negative potential applied to the suppressor grid, inducing current flow in the grid. By removing the dependence on beam energy and beam current a normalized suppressor grid current function can be formed which is dependent only on the gas line density. With this technique it is possible to infer the gas line density on a shot by shot basis.

\section{INTRODUCTION}

The neutral beam ion sources used at DIII-D consist of a plasma generator (called the arc chamber) and a four grid accelerator, Fig. 1. Gas is injected into the arc chamber and ionized, and then the positive ions are accelerated through a potential gradient to form an ion beam. For a beam energy of $80 \mathrm{keV}$ the grid potentials for the plasma, gradient, suppressor, and exit grids are $80 \mathrm{kV}, 63 \mathrm{kV},-3 \mathrm{kV}$, and $0 \mathrm{kV}$, respectively. A significant fraction of the gas injected into the arc chamber is not ionized into a plasma but flows through the accelerator into the neutralizer cell as neutral gas (called residual gas flow) where beam-gas interactions can ionize the gas.

A negative potential is applied to the suppressor grid to keep electrons produced outside the accelerator section from entering where they would be accelerated into the arc chamber. Slow positive ions produced in the neutralizer cell by beamgas interactions are attracted to the negative potential, and induce current flow in the grid. With a constant suppressor grid potential, the grid current depends on the quantity of neutral gas available for ionization, the number of beam particles per second passing through the gas (i.e. the beam current), and the velocity and species mix of the beam particles (which determines the cross section for slow ion production).

A normalized suppressor grid current, which is dependent only on the quantity of neutral gas in the neutralizer cell, is formed by removing the dependence on beam energy and beam current. Since the quantity of neutral gas in the neutralizer cell can be related to the line density via the measured neutralization efficiency, it is possible to obtain a

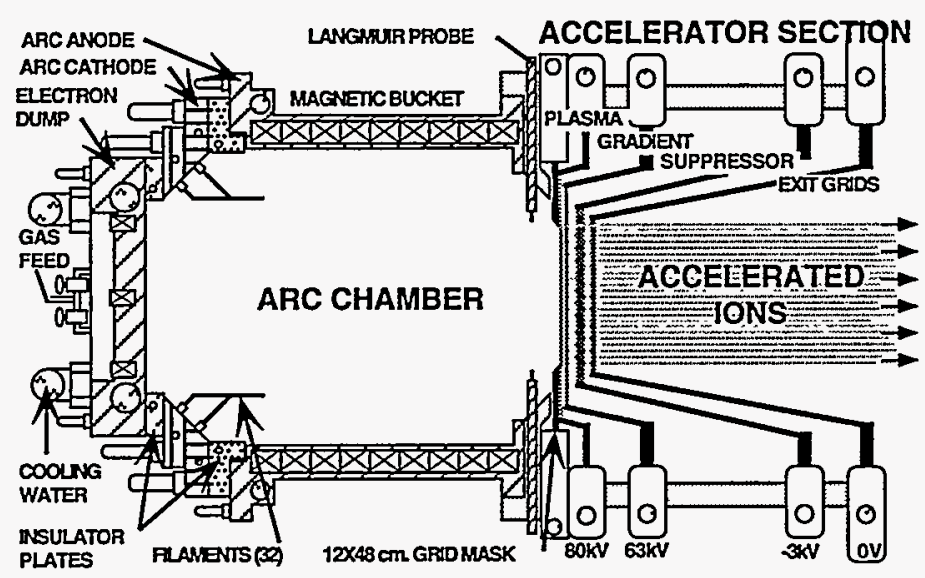

Fig. 1. A diagram of a DIII-D neutral beam positive ion source.

\footnotetext{
*Work supported by the U.S. Department of Energy under Contract No. DE-AC03-89ER51114.
} 
relationship between the (neutralizer cell) neutral gas line density and the normalized suppressor grid current.

To verify this concept we operated an ion source at various beam energies with different rates of gas-flow into the arc chamber and neutralizer cell. Measurements of the suppressor grid current and the neutralization efficiency of energetic ions, combined with theoretical calculations of the neutralization efficiency as a function of the gas line density, allowed us to obtain a relationship between the suppressor grid current and the neutral gas line density inside the neutralizer cell.

\section{THE RESIDUAL GAS FLOW FROM THE ARC CHAMBER INTO THE NEUTRALIZER CELL}

The first step in finding a relationship between the gas line density and the suppressor grid current is to obtain the residual gas flow rate from the arc chamber into the neutralizer cell. The residual gas flow rate is obtained by extrapolating the linear fit of the suppressor grid current vs. $1 / 2$ neutralizer gas flow rate to the $x$-intercept, Fig. 2 . The $x$-axis scaling factor of $1 / 2$ will be expalined below. We expect that the linear fit should intercept the $\mathrm{x}$-axis where the total gas flow rate (residual gas from the arc chamber plus neutralizer gas) equals zero since no suppressor grid current should be induced (here we ignore the very small amount of current resulting from beam ions striking the grid). An x-intercept of, say, minus eight torr $\ell / \mathrm{s}$ implies that the residual gas flow rate to the neutralizer cell is eight torr $\ell / s$ (i.e. that the $x$-axis scaling has been "shifted" by the unaccounted for residual arc chamber gas contribution).

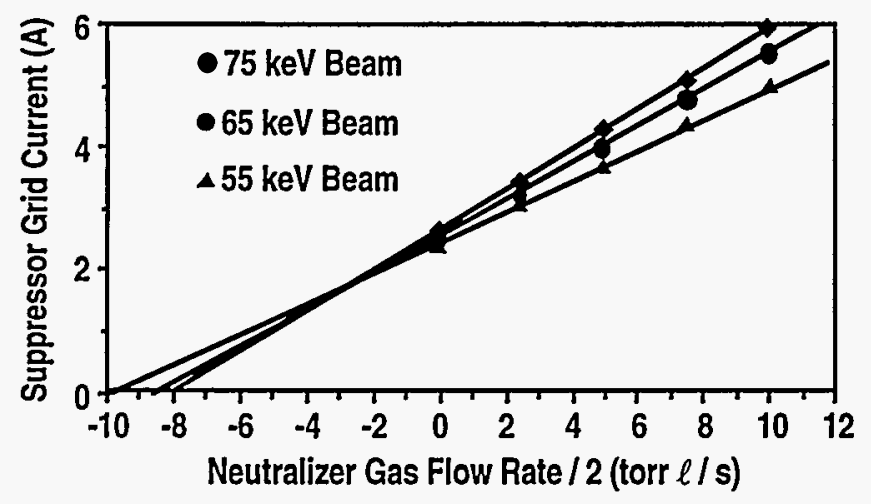

Fig. 2. The suppressor grid current plotted against one half of the neutralizer gas flow rate. The value between zero and an $x$-intercept provides the residual gas flow rate from the arc chamber into the neutralizer.

The gas from which slow ions are produced is comprised of residual gas flow from the arc chamber plus half the neutralizer gas flow. This can be seen from the slopes in Fig. 3 which show that the rate of increase in suppressor grid current due to changing neutralizer gas flow rate is half of that due to changing arc chamber gas flow rate. Since the beam current is constant, any extra arc chamber gas will exit
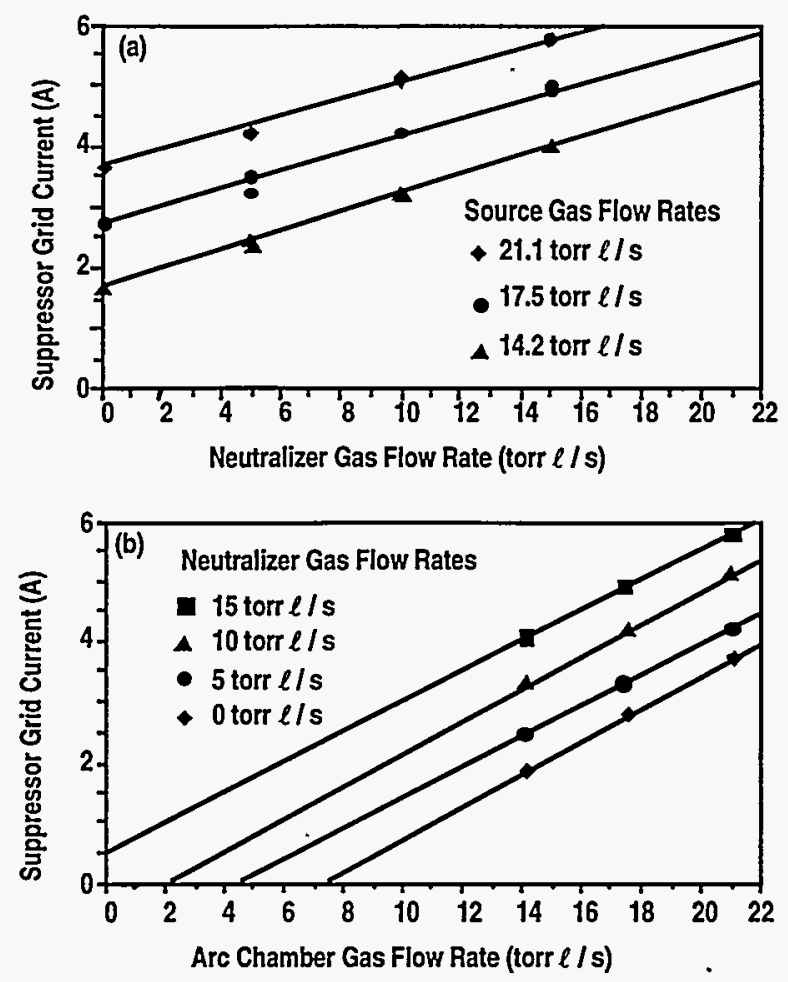

Fig. 3. (a) The suppressor grid current plotted against the neutralizer gas flow rate. (b) The suppressor grid current plotted against the arc chamber gas flow rate. Dividing a slope in plot $A$ by one in plot $B$ gives the fraction that the $\mathrm{X}$-axis in plot $\mathrm{A}$ scales (in regards to inducing suppressor grid current) compared to plot $B$.

and contribute to the gas available for ionization. Thus the arc chamber gas flow rate is the reference against which we can find the fraction of neutralizer gas flowing toward the accelerator region. This is the reason why the $\mathrm{x}$-axis in Fig. 2 is scaled at one half of the neutralizer gas flow rate.

The suppressor grid current is plotted against the arc chamber gas plus half the neutralizer gas flow rate (called the effective gas flow rate) in Fig. 4. The different slope for each beam energy results from the effects of differing beam current and ion production cross section.

\section{THE RELATIONSHIP BETWEEN THE NORMALIZED SUPPRESSOR GRID CURRENT AND THE EFFECTIVE GAS FLOW RATE}

It has been mentioned that the rate of ion production inside the neutralizer cell is dependent on the number of fast ions per second passing through the neutralizer cell , the cross section for ion production (which is dependent on the beam energy and ion species mix), and the quantity of neutral gas inside the neutralizer cell. Normalizing the suppressor grid current to the beam current and effective ionization cross section, $\Omega$ (i.e. $I_{\text {sup }}=I_{\text {sup }} / I_{\text {beam }} / \Omega$ ), we found that $I_{\text {sup }}$ is linearly proportional to the effective gas flow rate to the neutralizer cell, Fig. 5 . 


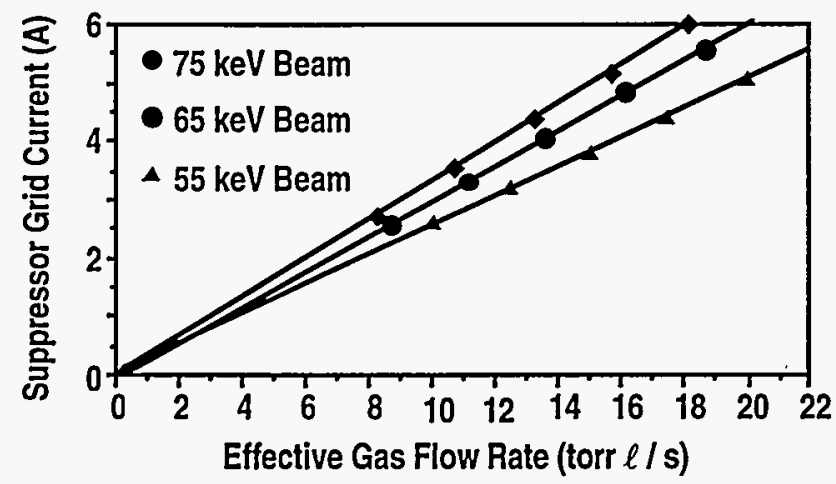

Fig. 4. The suppressor grid current plotted against the effective gas flow rate. The effect of differing beam current and ion production cross sections at each beam energy is evident in the different slopes.

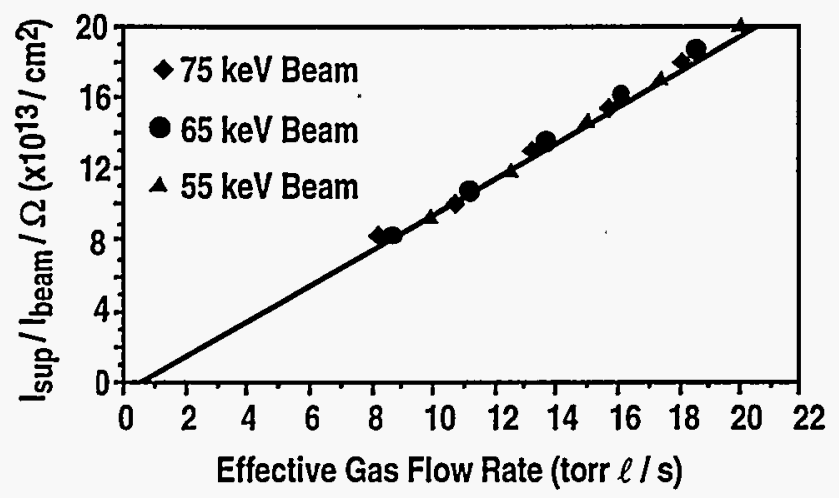

Fig. 5. The normalized suppressor grid current plotted against the effective gas flow rate shows that the normalized suppressor grid current is linearly proportional to the effective gas flow rate to the neutralizer cell and independent of other beam parameters.

\section{THE RELATIONSHIP BETWEEN THE EFFECTIVE GAS FLOW RATE, NEUTRALIZATION EFFICIENCY, AND NEUTRAL GAS LINE DENSITY}

In order to obtain the relationship between the normalized suppressor grid current and the gas line density, the effective gas flow rate must be related to the gas line density. Since the neutralization efficiency of the energetic ion beam can be experimentally measured as a function of the effective gas flow rate, its measurement, along with data relating the neutralization efficiency to the gas line density will yield the relationship between the effective gas flow rate and the gas line density.

Obtaining the neutralization efficiency as a function of the effective gas flow rate is accomplished by measuring the neutralization efficiency as a function of the neutralizer gas flow rate [1], and then changing the $x$-axis to be the effective gas flow rate (using the method described earlier). The neutralization efficiency is obtained from the ratio of power deposited on the beam dump by the neutralized energetic beam to that by the whole energetic beam and is shown in Fig. 6(a).
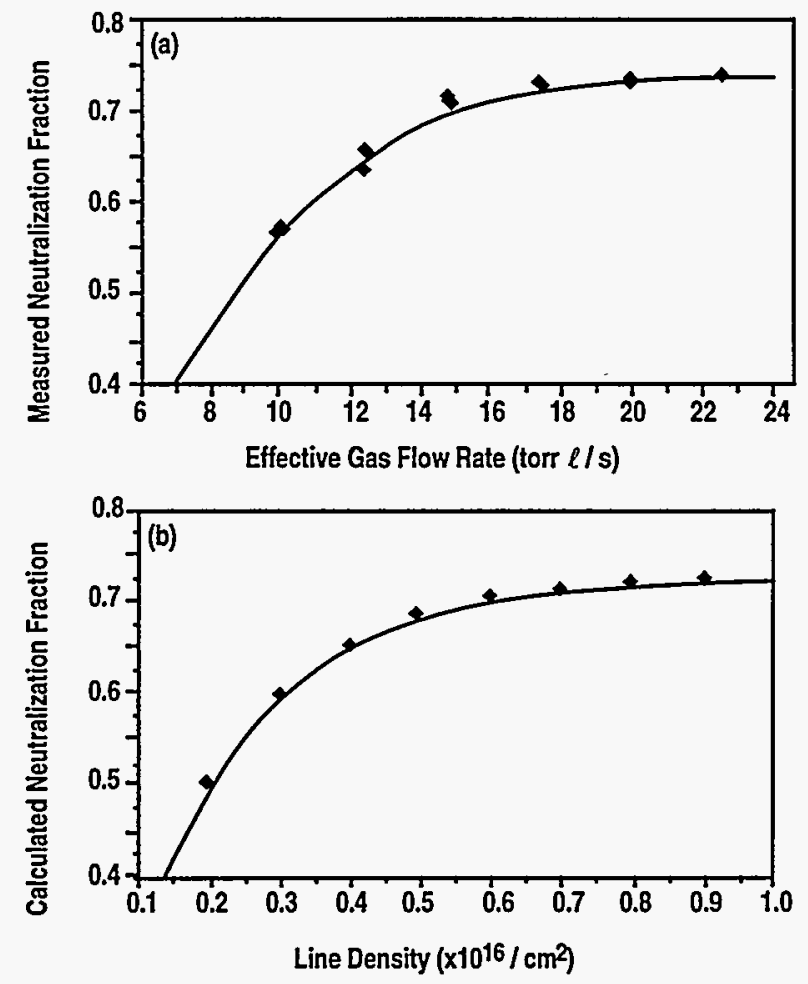

Fig. 6. (a) The measured neutralization fraction plotted against the effective gas flow rate. The data are for a $65 \mathrm{keV}$, 1.5 second beam. (b) The calculated neutralization fraction plotted against the neutral gas line density. The data in plot $B$ are from a mathematical model using the beam energy and measured species mix from the data in Plot A.

The neutralization fraction as a function of the gas line density, Fig. 6(b), is obtained from a computer program incorporating a mathematical model by Kim and Haselton, [2]. By combining the data in Figs. 6(a) and 6(b), the relationship between the gas line density and the effective gas flow rate is obtained and is plotted in Fig. 7. Note that the data in Fig. 7 was derived from $65 \mathrm{keV}$ data. We have plotted the gas line density as a function of the effective gas flow rate, including $55 \mathrm{keV}$ and $75 \mathrm{keV}$ data, and found that it overlays the $65 \mathrm{keV}$ data, indicating that the data in Fig. 7 is independent of the beam energy and beam current.

The neutral gas line density as a function of the effective gas flow rate (Fig. 7) is linear for lower effective gas flow rates, as expected, but deviates from linearity at higher gas flow rates (and higher gas line densities). This is because the asymptote in the measurement of the neutralization efficiency does not exactly agree with that from the model of the neutralization fraction as a function of the gas line density. Thus a small discrepancy between the two will result in a large uncertainty at high effective gas flow rates. For this reason we applied a linear fit to the data at the lower effective gas flow rates, and will use this line to represent the gas line density as a function of the effective gas flow rate. 


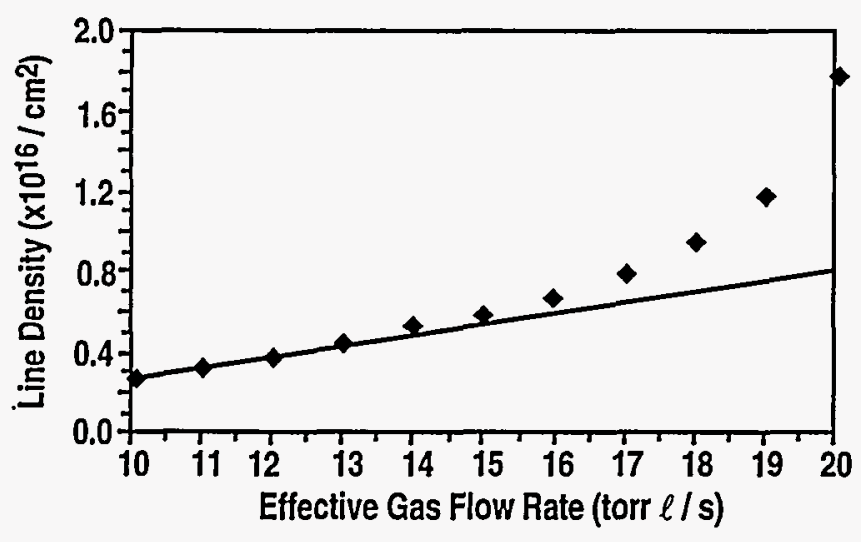

Fig. 7. The neutralizer gas line density plotted against the effective gas flow rate. The line density is linearly proportional to the effective gas flow rate for moderate gas flow rates.

\section{THE GAS LINE DENSITY AS A FUNCTION OF THE NORMALIZED SUPPRESSOR GRID CURRENT}

From the linear fits in Figs. 5 and 7 we obtain the neutralizer gas line density as a function of the normalized suppressor grid current and plot it in Fig. 8. Using this function we are able to infer the neutralizer gas line density based on the measured (and normalized) suppressor grid current. This allows us to adjust the neutralizer gas flow for sufficient gas line density to achieve optimum neutralization efficiency of the energetic ion beam, and thus provide maximum neutral beam power.

\section{CONCLUSION}

We have presented a new technique to infer the neutral gas line density inside the neutralizer cell of the DIII-D neutral beam system on a shot by shot basis. In accomplishing this we showed that the suppressor grid current (at constant suppressor potential) can be normalized so that it is independent

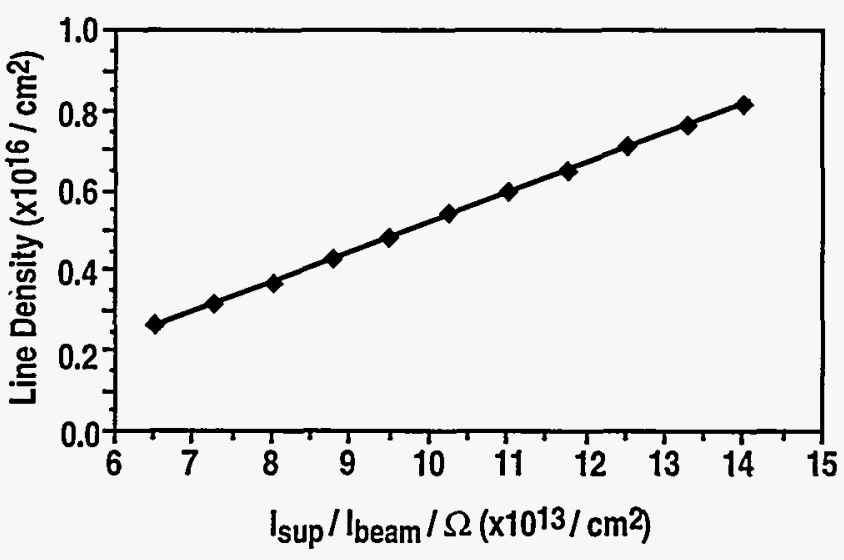

Fig. 8. The neutralizer gas line density plotted against the normalized suppressor grid current.

of all beam parameters except the total neutral gas flow rate to the neutralizer; and that the normalized suppressor grid current, since it is dependent on the quantity of neutral gas in the neutralizer cell, can be related to the neutral gas line density.

\section{ACKNOWLEDGMENT}

The authors would like to thank Bonnie Scoville and Matt Madruga for the operation of the neutral beam systems which supplied the data used in this paper.

\section{REFERENCES}

[1] D.N. Kessler, R.-M. Hong, D.H. Kellman, "Investigation of collisional effects within the bending magnet region of a DIII-D neutral beamline," Proc. 15th IEEE/NPSS Symp. on Fusion Engineering, pp. 427-430, 1993.

[2] J. Kim and H.H. Haselton, "Analysis of particle species evolution in neutral-beam injection lines," J. Appl. Phys. 50, p. 3802, 1979 